\title{
Vom Diskussionsforum zur Keimzelle alternativer Praxis in der Justiz? Bericht über den 7. Richterratschlag
}

Das Jahr 1983 bescherte Teilen der bundesdeutschen Justizjuristen bisher nicht gekannte öffentliche Aufmerksamkeit. Sie entstand wegen der vielfältigen friedenspolitischen Aktivitäten der »Richter und Staatsanwälte für den Frieden « und den sich daraus teilweise entwickelnden Konflikten mit Disziplinarvorgesetzten. ${ }^{1}$ Schlagzeilen machte aber auch die Auseinandersetzung der Fa. Hoechst AG mit Richtern der hessischen Arbeitsgerichtsbarkeit."

Die friedenspolitischen Aktivitäten der Richter und Staatsanwälte wären ohne das gemeinsame Diskussions- und Planungsforum des 6. Richterratschlags nicht realisierbar gewesen. ${ }^{3}$ Der intensive Kontakt der engagierten Richter trug wesentlich dazu bei, daß die besonders in die Schußlinie von wirtschaftlichen Machtgruppen oder auch von Dienstvorgesetzten geratenen Richter sich stets einer überregionalen Solidarität vieler Kolleginnen und Kollegen sicher sein konnten. Wer den Anpassungsdruck in der Justiz kennt und sich die massiven Angriffe auf einzelne Richter z. B. der Arbeitsgerichtsbarkeit in Frankfurt vor Augen führt, weiß, wie wichtig solche Unterstützung ist. Ohne sie ist in der Regel der einzelne völlig machtlos.

Die mit der Gründung des Richterratschlags vor vier Jahren beabsichtigte doppelte Zielsetzung, die gesellschaftspolitische Diskussion unter den Justizjuristen zu intensivieren und die wegen ihres Engagements oder ihrer Rechtsprechung angegriffenen Kolleginnen und Kollegen zu unterstützen, hat sich damit im vergangenen Jahr auch öffentlich sichtbar erfüllt. Der Richterratschlag hat sich zu einer Institution entwikkelt, von der (gerade angesichts der politischen Wende in diesem Land) weitere Impulse für eine nicht angepaßte Haltung innerhalb und außerhalb der Justiz zu erwarten sind.

Dies bestätigte auch der Verlauf des 7. Richterratschlags vom I 3. bis I s. I. 1984. Das Thema "Alle Staatsgewalt geht vom Volke aus - Ziviler Ungehorsam* lockte 193 Richter und Staatsanwälte aus dem ganzen Bundesgebiet in die Tagungsstätte des Gustav-Stresemann-Instituts nach Bad Bevensen. Wie immer stand die persönliche Betroffenheit im Mittelpunkt der Diskussionen; ebenso wichtig wie die gemeinsame "Arbeit« war aber auch dieses Mal die Freude am Zusammensein. Das von der Hamburger Vorbereitungsgruppe erstellte »Kulturprogramm« leistete hierzu einen großen Beitrag.

Nach einem dem persönlichen Kennenlernen bzw. dem Wiedersehen vorbehaltenen ersten Abend führte Günter Frankenberg am Samstag zunächst in die allgemeine

I Vgl. z. B. den Aufruf des 1. Forums Richter und Staatsanwälte für den Frieden an die BundestagsAbgeordneten, in: FR vom 6.6.1983.

$2 \mathrm{Vgl}$. den Beitrag von R. Erd in diesem Heft S. $58 \mathrm{ff}$.

3 Siehe Beschluß des 6. Richterratschlages in Gießen, abgedruckt in: KJ 1983, S. 229. 
Problematik des "Zivilen Ungehorsams praktischen wie theoretischen Wurzeln der Diskussion um den Zivilen Ungehorsam hin. Besondere Bedeutung maß er in diesem Zusammenhang der amerikanischen Bürgerrechtsbewegung und dem auf ihr aufbauenden grundlegenden Buch von J. Rawls", "Theorie der Gerechtigkeit", bei. In seiner Auseinandersetzung mit der neueren deutschen Literatur zu diesem Thema ${ }^{6}$ machte er deutlich, daß er eine direkte Einbeziehung des Zivilen Ungehorsams als Rechtsinstitut in das bestehende Legalsystem ablehnt. Eine Verortung des Zivilen Ungehorsams z. B. im Grundrechtssystem führe entweder zur Verwischung der Distanz zum Widerstandsrecht nach Art. 20 Abs. 4 GG oder zur Aufhebung der Grenzen grundrechtlich erlaubter Handlungen.

Konstitutiver Bestandteil des Zivilen Ungehorsams sei u. a. die begrenzte, gewaltfreie und verhältnismäßlige Regelverletzung, gleichzeitig aber auch die Inkaufnahme staatlicher Sanktionen. Letztere hätten sich in ihrer Schwere an den berechtigten und uneigennützigen Motiven der Akteure zu or:entieren. Nach Frankenberg ist das Institut des Zivilen Ungehorsams im Spannungsverhältnis von Volkssouveränität, Minderheitenschutz und Demokratie anzusiedeln. Dies entzieht es einer direkten Justiziabilität im Rahmen von Tatbestands- und Rechtswidrigkeitserörterungen, wohingegen es bei der Strafzumessung zu berücksichtigen ist. Zugleich weist der Zivile Ungehorsam als eine Theorie materieller Gerechtigkeit in die Zukunft eines Rechts der postbürgerlichen Gesellschaft, wie in der Diskussion herausgearbeitet wurde.

Trotz eines gewissen Bedauerns über die Ausklammerung des Gewaltbegriffs in seiner spezifisch deutschen Ausprägung bestand mit dem Referenten weitgehende Einigkeit darüber, daß im Falle objektiver und subjektiver Regelverletzung die Berufung auf Zivilen Ungehorsam keine unmittelbar strafbefreiende Wirkung haben kann, wohl aber zur Relativierung der strafrechtlichen Beurteilung der Tat führen muß. Allerdings war man sich auch darüber einig, daß in jedem Falle zunächst mit den klassischen juristischen Mitteln zu untersuchen ist, ob überhaupt eine Rechtsverletzung vorliegt. Für symbolische Sitzblockaden werde dies hinsichtlich des Nötigungstatbestands deutlich verneint. Ein Rekurs auf Zivilen Ungehorsam kann und muß dann unterbleiben.

Die nachfolgende Arbeit in Gruppen hatte sodann wie immer sehr konkrete, die eigene Arbeit betreffende Themenstellungen. Es wurde u. a. über Protest und Widerstandsformen im Arbeitsleben, über Möglichkeiten und Bedeutung eigner Aktionen von Richtern und Staatsanwälten, über Disziplinierungsversuche, bisher wenig bekannte Protestformen und über die rechtlichen Aspekte von Sitzblockaden diskutiert. In zwei vorbereitenden Broschüren lagen Stellungnahmen von Teilnehmern und Dokumente zu allen Themenbereichen vor. Allerdings kam man in den Diskussionsrunden zu wenig auf diese schriftlichen Vorlagen zurück. Dies hatte freilich den Vorteil, daß sich die Beiträge nicht zu sehr auf diejenigen konzentrierten, die sich bereits schriftlich geäußert hatten.

In der Arbeitsgruppe "Widerstand im Arbeitsleben" nahm der Fall Krauss gegen Hoechst AG und die sich daran anschließende Auseinandersetzung des Unterneh-

4 Die Tatsache, daß hier erstmals ein »justizfremder * Jurist einen rechtlichen Beitrag auf dem Richterratschlag hielt, war im Vorfeld nicht ganz unproblematisch, grundsätzlich sollen auch in Zukunft die Beiträge von Teilnehmern kommen.

s Frankfurt am Main 1979.

6 E. Küchenhoff, Ziviler Ungehorsam als Rechtsinstitut, Vorgänge Heft 62/63, 1983, S. 1 40 ff.; R. Dreier, Widerstandsrecht im Rechtsstaat?, in: Recht und Staat im sozialen Wandel, Festschrift für Scupin, Berlin 1983, S. 573 ff.; R. Scholz, Rechtsfrieden im Rechtsstaat, NJW ${ }_{198}$, S. 705 ff.; vgl. im einzelnen dazu G. rsankenberg, JZ $198_{4}$, H. 4 . 
mens mit der Frankfurter Arbeitsgerichtsbarkeit breiten Raum ein. Die chronologische Darstellung des Falles und die Art der Intervention des Chemiekonzerns, die in dem berüchtigten Rechtsbeugegutachten von Prof. Geerds und in der Forderung nach Nichtteilnahme an einem ÖTV-Arbeitskreis gipfelte, ließ allen Teilnehmern den exemplarischen Charakter dieser Auseinandersetzung für den eigenen Arbeitsalltag deutlich werden. Angesichts des dem Chemiekonzern zur Verfügung stehenden Mobilisierungspotentials ${ }^{7}$ war die uneingeschränkte Solidarität für den betroffenen Kollegen besonders wichtig. Keiner der Anwesenden, auch nicht die etwa 30-40 Prozent nicht in der ÖTV organisierten Richter und Staatsanwälte, hielt die Mitgliedschaft im Arbeitskreis Arbeitsrecht der ÖTV für unvereinbar mit der Tätigkeit als Arbeitsrichter.

In mehreren anderen Arbeitsgruppen wurde über Möglichkeiten weiterer Aktivitäten von Richtern und Staatsanwälten in der Friedensbewegung wie in der Justiz selbst diskutiert. Hierbei ging es im wesentlichen um die Veranstaltung eines zweiten Friedensforums, um eine Unterstützung der Heilbronner Erklärung von Schriftstellern, um ein Informationsblatt des Richterratschlags für Justizjuristen sowie um weitere Möglichkeiten der Verbreiterung und Stabilisierung des Zusammenhalts im Richterratschlag. Besonders von Richtern aus ländlichen Gegenden, die ihre Vereinzelung beklagten, wurde auf die Bedeutung des Richterratschlags hingewiesen. Im Gegensatz zu den sechziger Jahren, als einige Richter in exponierter Stellung eine Reform »von oben « versuchten und damit scheiterten, bietet der Richterratschlag als solidarische »Basisbewegung « eher die Chance, auch abweichenden Meinungen und Haltungen in der Justiz längerfristig Gehör zu verschaffen.

Zur Verbesserung der Kommunikation untereinander wurde die Herausgabe eines Informationsblattes vorgeschlagen, das in lockerer Erscheinungsweise und Aufmachung Justizjuristen über die Richterratschläge, regionale Aktivitäten und andere justizrelevante Ereignisse informieren soll. Eine Planungsgruppe soll beim nächsten Richterratschlag, der vom I4. bis I6. September bei Nürnberg stattfindet ${ }^{8}$, ein Konzept vorlegen.

Die Frage eines zweiten Forums "Richter und Staatsanwälte für den Frieden « wurde unter dem Eindruck der begonnenen Stationierung und des dazu ergangenen Beschlusses des Bundesverfassungsgerichts zunächst etwas zurückhaltend diskutiert. Nachdem man sich aber über die Gründe der gegenwärtig allenthalten in der Friedensbewegung anzutreffenden resignativen Tendenzen verständigt hatte, bestand rasch Einigkeit darüber, daß man gerade jetzt weiter aktiv bleiben müsse. Über das Was und Wie und vor allem über etwaige Organisatoren eines zweiten Forums gab es allerdings mehr offene Fragen als Antworten. Schließlich einigte man sich darauf, eine kritische Stellungnahme zum Beschluß des Bundesverfassungsgerichts zu erstellen und zu veröffentlichen.

Im übrigen wurde ein zweites Forum »Richter und Staatsanwälte für den Frieden « für Ende 1984 /Anfang 1985 geplant. Zwei Themenvorschläge standen dabei im Vordergrund: Die Möglichkeiten einer europäischen Friedensordnung, wobei insbesondere auf die deutsche Lage nach Jalta, die Ursachen und Folgen des Fehlens eines Friedensvertrags und auf historische wie gegenwärtige alternative Lösungsansätze eingegangen werden sollte. Der andere, zunächst zurückgestellte Vorschlag hatte

$7 \mathrm{Vgl}$. nur beispielhaft F. Wassner, in: FAZ vom 2. 12.1982 und 22. 10.83, Gehrmann, in: Die Zeit vom 25. Ir. I983, sowie Ch. Berglar, Syndikus der Höchst AG - dies Abhängigkeitsverhältnis jedoch tunlichst unterschlagend -, in: ZRP i $984, \mathrm{~S}_{4} \mathrm{ff}$.

8 Thema des 8. Richterratschlags: Justiz I984: Automatisierung und Recht. Rationalisierung der Gerichte Rationierung der Gerechtigkeit. Anmeldung möglichst bis 20. 5.84 an Sophie Ballestrem, Anna-DandlerStr. 9, 8 München 60 (Thema ist auch Datenschutz. Volkszählung, Personalausweis). 
eine mehr demokratietheoretische Ausrichtung. Er sah vor, Fragen wie »Mehrheitssystem und demokratische Legitimation «, "Repräsentativsystem und Kontrolle militärischer und wirtschaftlicher Macht« oder der "Zusammenhang zwischen Gewaltmonopol des Staates und seiner Schutzpflicht gegenüber den Bürgern« zu erörtern. ${ }^{9} \mathrm{Ob}$ ein derartiges zweites Forum tatsächlich stattfinden wird, dürfte wesentlich davon abhängen, ob sich eine Arbeitsgruppe findet, die die planerischen und organisatorischen Aufgaben übernimmt.

Kontroverse Stellungnahmen löste vor allem die Diskussion über die »Heilbronner Erklärung einiger Schriftsteller aus. Dabei bestand von Anfang an Übereinstimmung, den als mißlungen empfundenen Text nicht zu unterzeichnen, aber die Autoren gegenüber den juristisch unhaltbaren Angriffen aus den Reihen von CDU/CSU, FDP und SPD in Schutz zu nehmen.

Die Diskussion über Form und Inhalt der Stellungnahme berührte das Selbstverständnis der Teilnehmer des Richterratschlags. Denn es war nur eine kleine Gruppe von zuletzt vier bis fünf Richtern, die sich mit einem Teil des vorgeschlagenen Textes nicht einverstanden erklären wollte. Es handelte sich dabei um in ihrer Rechtsprechung betroffene Verwaltungsrichter. Bei allem Verständnis für ihre Sorge, potentielle Verweigerer auf einen "falschen « Weg zu führen, schienen sie den meisten auch der Überzahl der anwesenden Verwaltungsrichter - doch die Rechtsprechung zur sog. politisch motivierten Gewissensentscheidung allzusehr internalisiert zu haben. Letztlich wurde aber der nicht konsensfähige Passus gestrichen - eine Lösung, die zwar die Situation entkrampfte, aber keine allgemeine Befriedigung hinterließ. Die Frage nach dem Umgang mit Minderheitspositionen wird den Richterratschlag sicherlich noch weiter beschäftigen.

Mehr noch als früher lag beim 7. Richterratschlag einer der Höhepunkte in der Gestaltung der Freizeit. Hamburger Richter und Staatsanwälte hatten in monatelangen Proben das Stück "Die Neun von Catonsville« von Daniel Berrigan eingeübt. Die Darbietung am Samstag Abend wurde zu einem großen Erfolg, vielleicht sogar zur Geburtsstunde eines neuen Ensembles; zumindest ließ sich das aus den Bitten um weitere Vorführungen an anderen Orten schließen. Eindringlicher kann Richtern und Staatsanwälten die Ausblendung wesentlicher Tatmotive bei der "Wahrheitsfindung " im Prozeß kaum vor Augen geführt werden, als dies in dem Stück geschah. Betroffenes Schweigen und anschließend minutenlanger Applaus für die schauspielernden Kolleginnen und Kollegen war der Dank der Zuschauer. Es wäre gut, war allenthalben zu hören, wenn jeder Richter und Staatsanwalt diesen Spiegel des öfteren in seinem Berufsleben vorgehalten bekäme. Gemeinsam gesungene Ratschläge aus dem Liederbuch des Richterratschlags und die ebenso lustigen wie bissigen Gesänge einer Moritatengruppe aus Frankfurt und Hamburg ließen den gelungenen Abend in eine lange Nacht übergehen.

Am anderen Morgen wurden die Ergebnisse der Arbeitsgruppen diskutiert und eine (im Anhang abgedruckte) Erklärung verabschiedet.

Hartmut Bäumer 
$\gg I$

I. Viele Menschen beschreiben ihr Opponieren gegen die Raketenstationierung als ,Widerstand oder >zivilen Ungehorsam‘. Dieses Selbstverständnis soll und darf man nicht durch öffentliche Sprachregelungen unterbinden. Die handelnden Menschen müssen selbst entscheiden, ob sie die drohenden Gefahren der weiteren Aufrüstung für so ernst halten, daß Widerstand und Ungehorsam am Platze sind. Die Risiken des Handelns trägt jeder selbst. Die Voraussetzungen des Widerstandsrechts gemäß Art. 20 Abs. 4 des Grundgesetzes liegen derzeit nicht vor.

Ein Widerstehen von Juristen in der Justiz kann aber gefordert sein, wenn es gilt, sich Zwängen und Zumutungen zu entziehen, wetche ein Handeln in richterlicher Unabhängigkeit erschweren.

Wir werden weiterhin bei der Rechtsprechung in voller Gesetzes- und Verfassungstreue die Spielräume, die das Recht allenthalben bietet, in fortschrittlicher und die Mitwirkung des Volkes in die Politik fördernder Weise nutzen. Dabei wollen wir unsere Erfahrungen und Arbeitsergebnisse füreinander nutzbar machen und austauschen.

2. Wir wollen auch an der Willensbildung des Volkes in der Frage der Rüstungsgefahren als Richter und Staatsanwälte teilnehmen. Die Disziplinierungsmaßnahmen durch Dienstvorgesetzte nehmen zu. Dagegen werden wir uns wehren. Die Art und Weise der Gegenwehr werden wir mit den jeweils Betroffenen diskutieren. Wir werden unsere Anschauungen zum Richterdienstrecht, zur Meinungsfreiheit und zu den politischen Sachfragen öffentlich vertreten, auch wenn im Einzelfall rechtliche Abwehr unnötig, unzweckmäßig oder erfolglos ist.

3. Widerstand und Abwehr von Disziplinarmaßnahmen können vom einzelnen nur schwer getragen werden. Die Mitglieder des Richterratschlags werden einander unterstützen.

4. Wir begrüßen es, daß nach dem Beginn der Stationierung von Pershing II und Cruise Missiles Schriftsteller in der Heilbronner Erklärung zu den Problemen Stellung genommen haben, die sich hieraus für den Dienst in der Bundeswehr ergeben können. Sie haben dabei von ihrem Grundrecht auf freie Meinungsäußerung Gebrauch gemacht; wir verurteilen die Versuche zur Kriminalisierung der Autoren. Wir erinnern in diesem Zusammenhang daran, daß 1958 der damalige Abgeordnete und spätere Bundespräsident Heinemann von einer Pflicht zur Gehorsamsverweigerung gesprochen hat, wenn es um Massenvernichtungsmittel geht.

5. Wir empfehlen die weitere Auseinandersetzung mit dem Thema, Wehrgerichtsbarkeit،. Nachdem es durch das erste Forum >Richter und Staatsanwälte für den Frieden bundesweit Aufmerksamkeit fand, wird es auf regionaler Ebene weiter vertieft werden.

II.

6. Wir halten es weiter für notwendig, der breiten Öffentlichkeit durch mannigfaltige Aktionen bewußt zu machen, daß angesichts des Ausmaßes der Bedrohung alle Zivilschutzbemühungen absurd sind.

7. Darüber hinaus begrüßen wir die Bestrebungen der Friedensinitiativen, Volksbefragungsaktionen zur Frage der Aufstellung der neuen Mittelstreckenraketen durchzuführen.

8. Für den Fall von Rechtsverletzungen sollte folgendes beachtet werden: Dient sie ausschließlich einem politischen oder staatsbürgerlichen Anliegen, das nach der Werteordnung des Grundgesetzes billigenswert erscheint, sollte sich die Schwere 
einer strafrechtlichen oder anderen staatlichen Sanktion an diesem Handlungsziel orientieren.

9. Bei der Bewertung auch unbekannter und verblüffender Aktionen muß der Grundsatz gelten: ,Es ist alles erlaubt, was nicht ausdrücklich verboten ist!

III

I0. Die unmittelbare Betroffenheit von Richtern mit Widerstandsaktionen im Arbeitsleben (zum Beispiel Protesten) ist noch gering.

I r. Ob und wie betriebliche Proteste sanktioniert werden, hängt vermutlich von den gewerkschaftlichen und politischen Rahmenbedingungen ab. Massive gewerkschaftliche Gegenwehr fördert die Bereitschaft des Arbeitgebers zum Einlenken, während Konflikte gewerkschaftlich isolierter Kollegen eher gerichtlich mit aller Härte ausgetragen werden.

12. In der neueren höchstrichterlichen Rechtsprechung ist eine bedenkliche Einschränkung des Grundrechts auf freie Meinungsäußerung festzustellen. Dies manifestiert sich zum Beispiel in Entscheidungen des Bundesarbeitsgerichts zur Frage des Plakettentragens im Betrieb.

13. Aufgabe der Rechtsprechung ist es demgegenüber nicht nur, den bisher erreichten Standard zu halten und weitere Verschiebungen zu Lasten von Arbeitnehmern zu verhindern, sondern darüber hinaus zum Beispiel durch konsequente Grundrechtsabwägungen die Freiheitsräume der Arbeitnehmer im Betrieb zu erweitern.

14. Die Teilnahme an gewerkschaftlichen Arbeitskreisen, in denen rechtliche und rechtspolitische Fragen unabhängig vom Einzelfall erörtert werden, ist ein geeignetes und zulässiges Mittel der gewerkschaftlichen Betätigung von Richtern. Dies gilt insbesondere auch für Richter an Arbeitsgerichten.

I5. Die Angriffe von Arbeitgebern und konservativer Presse gegen die Teilnahme von Arbeitsrichtern an derartigen Arbeitskreisen ist damit ein Angriff auf das Grundrecht der Koalitionsfreiheit, letztlich aber gegen das unerwünschte Bild eines politisch bewußt handelnden Richters." 